\title{
Existence of Solutions for some Elliptic Problems with Critical Sobolev Exponents
}

Mario Zuluaga

\section{Introduction}

Let $\Omega$ be a bounded domain in $\mathbb{R}^{n}$ with $n \geqslant 3$. In this paper we are concerned with the problem of finding $u \in H_{0}^{1}(\Omega)$ satisfying the nonlinear elliptic problems

$$
\Delta u+|u|^{\frac{n+2}{n-2}}+f(x)=0
$$

in $\Omega$ and $u(x)=0$ on $\partial \Omega$, and

$$
\Delta u+u+|u|^{\frac{n+2}{n-2}}+f(x)=0
$$

in $\Omega$ and $u(x)=0$ on $\partial \Omega$, when of $f \in L^{\infty}(\Omega)$.

The exponent $q=(n+2) /(n-2)$ is critical for the Sobolev embedding of $H_{0}^{1}(\Omega)$ in $L^{q+1}(\Omega)$. This embedding is not compact and therefore the operator $F: H_{0}^{1}(\Omega) \rightarrow H_{0}^{1}(\Omega)$, for which its fixed points are solutions of (1.1) or (1.2), is not compact. For these reasons, standard fixed-points methods can not be applied to find solutions of (1.1) and (1.2). In this paper we study (1.1) and (1.2) by making use of a fixed point theorem as well as one from approximation methods. Problems of type (1.1) and (1.2) have been studied in [2] and [4]. In these papers the authors find positive solutions. Their methods are variational and their work is related to the Yamabe Problem. For a complete description of the Problem of Yamabe, we refer to [6]. 
If $f=0$, it is well known that the equation (1.1) has no positive solutions. (See [2 p. 422].) If $f \neq 0$ we will see in Theorem 3.3 that the equation (1.1) has a nontrivial solution and in the case $f>0$, by the maximum principle, we have that the equation (1.1) has a positive solution.

Also, if $f=0$, the equation (1.2) has a positive solution in the case that $1 \in\left(0, \lambda_{1}\right)$, where $\lambda_{1}$ denote the first eigenvalue of $-\Delta$ with zero Dirichlet condition on $\Omega$. (See [2, p. 441].) If $f \neq 0$, we will see in Theorem 3.4 that equation (1.2) has nontrivial solution and that if $f>0$ then the solution is positive.

\section{Preliminaries}

Let $g: \Omega \times \mathbb{R} \rightarrow \mathbb{R}$ be defined by $g(x, u)=|u|^{s}+f$ in the case of problem (1.1), or $g(x, u)=u+|u|^{s}+f$, in the case of problem (1.2), and $0<s \leqslant(n+2) /(n-2)$. Then the operator of Nemytsky $G: L^{s+1}(\Omega) \rightarrow L^{\frac{s+1}{s}}(\Omega)$ defined by $G(u)(x)=$ $g(x, u(x))$ is continuous and bounded, so that for every $\epsilon>0$ there exists $r=r(\epsilon)$ such that if $\|u\|_{L^{s+1}(\Omega)} \leqslant r$, then $\|g(x, u)-g(x, 0)\|_{L^{(s+1) / s(\Omega)}} \leqslant \epsilon$ and we have the following inequality, (see [5, p. 26]),

$$
\|g(x, u)\|_{L^{(s+1) / s(\Omega)}} \leqslant\left[\left(\frac{\|u\|_{L^{s+1}(\Omega)}}{r}\right)^{s+1}+1\right]^{\frac{s}{s+1}} \epsilon+\|f\|_{L^{(s+1) / s(\Omega)}} .
$$

For short, we will indicate $\|\cdot\|_{L^{p(\Omega)}}=\|\cdot\|_{p}$, for all $p>0$.

It is well-known (see $[1$, p. 40]) that if $1 \leqslant s+1<2 n /(n-2), n \geqslant 3$, the inclusion $H_{0}^{1}(\Omega) \rightarrow L^{s+1}(\Omega)$ is completely continuous. If $1 \leqslant s+1 \leqslant 2 n /(n-2)$ the inclusion is only continuous and we have

$$
\|u\|_{s+1} \leqslant \hat{K}(s)\|u\|_{1,2},
$$

where $\|\cdot\|_{1,2}$ is the norm of the space $H_{0}^{1}(\Omega)$. In the case $s=1, \hat{K}(1)=1 / \sqrt{\lambda_{1}}$, where $\lambda_{1}$ is the first eigenvalue of the operator $-\Delta$.

If

$$
s=\frac{n+2}{n-2}
$$

then

$$
\hat{K}\left(\frac{n+2}{n-2}\right)=\frac{n-1}{n-2} \frac{1}{\sqrt{n}} .
$$

(see [1, p. 41]).

Since Measure $(\Omega)=|\Omega|$ is finite then

$$
\|u\|_{s+1} \leqslant|\Omega|^{\frac{1}{s+1}-\frac{n-2}{2 n}} \cdot\|u\|_{\frac{2 n}{n-2}} .
$$


(2.2) - (2.4) yield, for all $u \in H_{0}^{1}(\Omega)$,

$$
\|u\|_{s+1} \leqslant K(s)\|u\|_{1,2},
$$

where

$$
K(s)=\frac{1}{\sqrt{n}} \frac{n-1}{n-2}|\Omega|^{\frac{1}{s+1}}-\frac{n-2}{2 n}
$$

Solutions of (1.1) AND (1.2). Let

$$
g(x, u)=|u|^{s}+f \quad \text { or } \quad g(x, u)=|u|^{s}+u+f, \quad s \leqslant \frac{n+2}{n-2} .
$$

We say that $u \in H_{0}^{1}(\Omega)$ is a weak solution of (1.1) and (1.2) respectively, if for all $v \in H_{0}^{1}(\Omega)$

$$
\langle u, v\rangle_{1,2}=\int_{\Omega} g(x, u(x)) v(x) d x .
$$

For $u \in H_{0}^{1}(\Omega)$ fixed, the right side of (2.6) defines a linear, continuous functional. Then by Riesz's Theorem there exists $F: H_{0}^{1}(\Omega) \rightarrow H_{0}^{1}(\Omega)$ such that, for all $v \in H_{0}^{1}(\Omega)$,

$$
\langle F(u), v\rangle_{1,2}=\int_{\Omega} g(x, u) v
$$

Then, by virtue of (2.6) and (2.7), $u \in H_{0}^{1}(\Omega)$ is a weak solution of (1.1) or (1.2) if and only if $u$ is a fixed point of $F$. It is well-known that only for $s<(n+2) /(n-2), F$ is completely continuous. Our main tool will be the following Theorem due to Krasnosel'skii.

Theorem 2.1. Let $F: H \rightarrow H$ be a completely continuous operator defined on a Hilbert space $H$. Let $D \subset H$ be an open and bounded set such that $0 \notin \partial D$. Suppose that for all $u \in \partial D,\langle F(u), u\rangle \leqslant\|u\|^{2}$. Then $F$ has a fixed point in $\bar{D}$.

Proof. See [1, p. 271].

\section{The Main Results}

First, we are concerned with the following general problem

$$
\left.\begin{array}{rlr}
\Delta u+g(x, u)=0 & \text { in } & \Omega, \\
u(x)=0 & \text { on } & \partial \Omega,
\end{array}\right\}
$$


where $g: \bar{\Omega} \times \mathbb{R} \rightarrow \mathbb{R}$ is measurable in $x$, continuous in $u$ and satisfies

$$
|g(x, u)| \leqslant a(x)+b|u|^{s},
$$

where $a(x) \in L^{\frac{s+1}{s}}(\Omega), b>0,1<s+1<2 n /(n-2)$ and $n \geqslant 3$. Then we have

Theorem 3.1. Suppose that $g(x, u)$ satisfies (3.2) and $0<s<1$, then (3.1) has a weak solution.

Proof. In the same way as (2.1) we have

$$
\|g(x, u)\|_{\frac{s+1}{s}} \leqslant\left[\left(\frac{\|u\|_{s+1}}{r}\right)^{s+1}+1\right]^{\frac{s}{s+1}} \epsilon+\|g(x, 0)\|_{\frac{s+1}{s}} .
$$

(2.5), (2.7) and (3.3) give

$$
\langle F(u), u\rangle_{1,2} \leqslant\left\{\left[\left(\frac{K}{r}\|u\|_{1,2}\right)^{s+1}+1\right]^{\frac{s}{s+1}} \epsilon+\|g(x, 0)\|_{\frac{s+1}{s}}\right\} K\|u\|_{1,2} .
$$

We claim that there exists $y>0$ such that for $\|u\|_{1,2}=y$

$$
\left\{\left[\left(\frac{K}{r} y\right)^{s+1}+1\right]^{\frac{s}{s+1}} \epsilon+\|g(x, 0)\|_{\frac{s+1}{s}}\right\} K y \leqslant y^{2} .
$$

Since $0<s<1$ it is easy to see that, for $y>0$ sufficiently large, we get (3.5). For (3.4) and (3.5) we have that $\langle F(u), u\rangle_{1,2} \leqslant\|u\|_{1,2}^{2}$, where $\|u\|_{1,2}$ is sufficiently large. Then Theorem 3.1 follows from Theorem 2.1 .

Remark. Theorem 3.1 is a consequence of Theorem 2.5 in [3] (see Example 2.9, p. 122). There, Example 2.9 is done from a variational point of view.

Theorem 3.2. Suppose that $g(x, u)$ satisfies (3.2) and $s=1$. Then (3.1) has $a$ weak solution if $\lambda_{1}>b$.

Proof. If $\|u\|_{2} \leqslant r$ then $\|g(x, u)\|_{2} \leqslant\|a(x)\|_{2}+b r$. Let $\epsilon=\|a(x)\|_{2}+b r$. (3.5) yields

$$
\left(K^{2} y^{2}+r^{2}\right)^{1 / 2} \frac{\epsilon}{r}+\|g(x, 0)\|_{2} \leqslant \frac{y}{K} .
$$

It is clear that there exists $y>0$ such that $y$ satisfies (3.6) if

$$
\frac{1}{K^{2}}>K^{2} \frac{\epsilon^{2}}{r^{2}} \text {. }
$$


In this case, we know that $K^{2}=\frac{1}{\lambda_{1}}$, therefore (3.7) is equivalent to

$$
\lambda_{1}>\frac{\|a(x)\|_{2}}{r}+b
$$

For $r$ sufficiently large we get (3.8). Theorem 2.1 now implies our result.

Now we will return to our main problems (1.1) and (1.2). We have the following

Theorem 3.3. Assume that $f \in L^{\infty}(\Omega)$. Suppose that at least one of the following inequalities holds

$$
\|f\|_{\infty}<B(n)|\Omega|^{-\frac{n+2}{2 n}}, \text { if }|\Omega|>1,
$$

or

$$
\|f\|_{\infty}<B(n), \quad \text { if } \quad|\Omega| \leqslant 1
$$

where

$$
B(n)=\frac{\frac{4}{n+2}\left(\frac{n-2}{n+2}\right)^{\frac{n-2}{4}}}{\left(\frac{n-1}{n-2} \frac{1}{\sqrt{n}}\right)^{\frac{n+2}{2}}}, \quad n \geqslant 3 .
$$

Then problem (1.1) has at least a weak solution if we assume that $\partial \Omega$ is sufficiently smooth.

Proof. First we will consider the following problem

$$
\left.\begin{array}{rlc}
\Delta u+|u|^{s}+f=0 & \text { in } & \Omega, \\
u(x)=0 & \text { on } & \partial \Omega,
\end{array}\right\}
$$

and $1<s<\frac{n+2}{n-2}$.

Let $g(x, u)=|u|^{s}+f$. As in Theorems 3.1 and 3.2, the problem (3.11) has a weak solution $u_{s} \in H_{0}^{1}(\Omega)$ if there exists $y>0$ such that

$$
\left[\left(\frac{K}{r} y\right)^{s+1}+1\right]^{\frac{s}{s+1}} \epsilon+\|f\|_{\frac{s+1}{s}} \leqslant \frac{y}{K}
$$

where $K$ is the constant in (2.5). In this case, it is easy to see that $r=r(\epsilon)=\epsilon^{1 / s}$. 
Therefore (3.12) becomes

$$
\left[\left(K^{2} x\right)^{s+1}+\epsilon^{\frac{s+1}{s}}\right]^{\frac{s}{s+1}}+\|f\|_{\frac{s+1}{s}} \leqslant x,
$$

where $x=y / K$.

Since we can take $\epsilon$ sufficiently small, (3.13) has a solution $x>0$ if

$$
\left(K^{2} x\right)^{s}+\|f\|_{\frac{s+1}{s}}<x
$$

has a solution $x>0$.

Now, it is easy to see that (3.14) has the solution

$$
x_{0}=\left(\frac{1}{s K^{2 s}}\right)^{\frac{1}{s-1}}
$$

if

$$
\|f\|_{\frac{s+1}{s}}<\frac{\left(\frac{1}{s}\right)^{\frac{1}{s-1}}-\left(\frac{1}{s}\right)^{\frac{s}{s-1}}}{K^{\frac{2 s}{s-1}}}=\lambda(s) .
$$

Finally,

$$
\lim _{s \rightarrow \frac{n-2}{n-2}} \lambda(s)=B(n) .
$$

Also, for $s \in R$ such that $1<s<\frac{n+2}{n-2}$ we have

$$
\|f\|_{\frac{s+1}{s}} \leqslant\|f\|_{\infty}|\Omega|^{\frac{s}{s+1}} .
$$

Therefore, if $|\Omega|>1$, (3.9) yields

$$
\|f\|_{\frac{s+1}{s}} \leqslant\|f\|_{\infty}|\Omega|^{\frac{s}{s+1}}<\|f\|_{\infty}|\Omega|^{\frac{n+2}{2 n}}<B(n) .
$$

Or, if $|\Omega| \leqslant 1$, by (3.10) we have

$$
\|f\|_{\frac{s+1}{s}} \leqslant\|f\|_{\infty}<B(n) .
$$

Using (3.16), (3.17) and (3.18) we get that there exists $s_{0}$ such that $1<s_{0}<$ $\frac{n+2}{n-2}$ and, if $s \in\left(s_{0}, \frac{n+2}{n-2}\right)$, then (3.14) has a solution $x>0$ and therefore (3.11) has a weak solution.

Now, by Theorem 2.1 we have that for $s \in\left(s_{0}, \frac{n+2}{n-2}\right)$ the weak solution $u_{s} \in H_{0}^{1}(\Omega)$ of (3.11) satisfies,

$$
\left\|u_{s}\right\|_{1,2} \leqslant y_{0}=K x_{0}=\left(\frac{1}{s}\right)^{\frac{1}{s-1}} K^{\frac{1+s}{1-s}} .
$$


By (3.19) we obtain that for $s \in\left(s_{0}, \frac{n+2}{n-2}\right)$ the set $\left\{u_{s}\right\}$, such that $u_{s}$ is a weak solution of (3.11), is bounded. Then there exists $\left\{u_{k}\right\} \subset\left\{u_{s}\right\}$ such that

$$
W \lim _{k \rightarrow \frac{n+2}{n-2}} u_{k}=u_{\frac{n+2}{n-2}}
$$

for some $u_{\frac{n+2}{n-2}} \in H_{0}^{1}(\Omega)$. (Wlim indicates weak limit.)

For simplicity we will use $\frac{n+2}{n-2}=N$.

Our next step is to show that $u_{N}$ is a weak solution of (1.1).

Since $f \in L^{\infty}(\Omega)$, by an iterative argument called a bootstrapping procedure, (see [1, p. 50], we can see that $u_{s} \in H_{0}^{1}(\Omega)$, the weak solution of (3.11), satisfies that $u_{s} \in C^{0, \alpha}(\bar{\Omega})$, and since $\partial \Omega$ is sufficiently smooth, $u_{s}$ is, in particular, continuous on $\bar{\Omega}$.

As in (2.7) let $F_{s}: H_{0}^{1}(\Omega) \rightarrow H_{0}^{1}(\Omega)$ be defined as

$$
\left\langle F_{s}(u), v\right\rangle_{1,2}=\left\langle|u|^{s}+f, v\right\rangle_{2},
$$

for all $v \in H_{0}^{1}(\Omega)$.

For $s<N, F_{s}$ is completely continuous. As we saw, there exists $s_{0}<N$ such that for $s \in\left(s_{0}, N\right), F_{s}\left(u_{s}\right)=u_{s}$, where $u_{s}$ is a weak solution of (3.11).

Let $\left\{u_{k}\right\}$ a subsequence of $\left\{u_{s}\right\}, s \in\left(s_{0}, N\right)$, such that $u_{k} \rightarrow u_{N}$ if $k \rightarrow N$. ( $\rightarrow$ denotes weak convergence). By (3.21) we have

$$
\left\langle F_{k}\left(u_{k}\right), v\right\rangle_{1,2}=\left\langle u_{k}, v\right\rangle_{1,2}=\left\langle\left|u_{k}\right|^{k}+f, v\right\rangle_{2},
$$

for all $v \in H_{0}^{1}(\Omega)$. And therefore, for all $v \in H_{0}^{1}(\Omega)$

$$
\lim _{k \rightarrow N}\left\langle\left|u_{k}\right|^{k}+f, v\right\rangle_{2}=\left\langle u_{N}, v\right\rangle_{1,2} .
$$

On the other hand, $u_{k}, k \in\left(s_{0}, N\right)$, is continuous. Hence

$$
\left.\lim _{r \rightarrow N}|| u_{k}\right|^{r}-\left.\left|u_{k}\right|^{N}\right|^{\frac{2 n}{n+2}}=0,
$$

and by Lebesgue's dominated convergence Theorem we have that $\left|u_{k}\right|^{r} \rightarrow\left|u_{k}\right|^{N}$ in $L^{\frac{2 n}{n+2}}(\Omega)$ if $r \rightarrow N$. Now, for each $v \in H_{0}^{1}(\Omega)$ fixed, $\langle v, \bullet\rangle_{2}$ defines a linear and bounded functional on $L^{\frac{2 n}{n+2}}(\Omega)$. Hence, for all $v \in H_{0}^{1}(\Omega)$

$$
\lim _{r \rightarrow N}\left\langle\left|u_{k}\right|^{r}+f, v\right\rangle_{2}=\left\langle\left|u_{k}\right|^{N}+f, v\right\rangle_{2} .
$$

Also, $\left\{u_{k}\right\} \subset H_{0}^{1}(\Omega)$ is bounded, and since $H_{0}^{1}(\Omega)$ is embedded in $L^{\frac{2 n}{n+2}}(\Omega),\left\{u_{k}\right\}$ is bounded in $L^{\frac{2 n}{n+2}}(\Omega)$ as well. Furthermore the Nemytsky operator defined by $|u|^{N}$ is bounded, so that $\left\{\left|u_{k}\right|^{N}\right\}$ is bounded in $L^{\frac{2 n}{n+2}}(\Omega)$. Therefore, there exists 
$h \epsilon_{L^{2 n /(n+2)}}(\Omega)$ and a subsequence of $\left\{u_{k}\right\}$, labeled in the same form, such that for all $v \in H_{0}^{1}(\Omega)$

$$
\lim _{k \rightarrow N}\left\langle\left|u_{k}\right|^{N}+f, v\right\rangle_{2}=\langle h+f, v\rangle_{2}
$$

By (3.24) and (3.25) we get

$$
\lim _{r, k \rightarrow N}\left\langle\left|u_{k}\right|^{r}+f, v\right\rangle_{2}=\langle h+f, v\rangle_{2},
$$

for each $v \in H_{0}^{1}(\Omega)$. (3.23) and (3.26) yield

$$
\langle h+f, v\rangle_{2}=\left\langle u_{N}, v\right\rangle_{1,2},
$$

for each $v \in H_{0}^{1}(\Omega)$.

Also, since $u_{k} \rightarrow u_{N}$ in $H_{0}^{1}(\Omega)$, we have

$$
\lim _{k \rightarrow N}\left\langle\left|u_{k}\right|^{r}+f, v\right\rangle_{2}=\left\langle\left|u_{N}\right|^{r}+f, v\right\rangle_{2},
$$

for all $v \in H_{0}^{1}(\Omega)$, and $r<N$. (3.23), (3.26), (3.27) and (3.28) yield

$$
\lim _{r \rightarrow N}\left\langle\left|u_{N}\right|^{r}+f, v\right\rangle_{2}=\langle h+f, v\rangle_{2},
$$

for each $v \in H_{0}^{1}(\Omega)$. Since $\left|u_{N}\right|^{r} v \leqslant\left(\left|u_{N}\right|^{N}+1\right)|v|$, by Lebesgue's dominated convergence Theorem we have

$$
\lim _{r \rightarrow N}\left\langle\left|u_{N}\right|^{r}+f, v\right\rangle_{2}=\left\langle\left|u_{N}\right|^{N}+f, v\right\rangle_{2},
$$

for each $v \in H_{0}^{1}(\Omega)$. (3.30) and (3.29) yield

$$
\left\langle\left|u_{N}\right|^{N}+f, v\right\rangle_{2}=\left\langle u_{N}, v\right\rangle_{1,2},
$$

for each $v \in H_{0}^{1}(\Omega)$. By (3.31) we have that $u_{N} \in H_{0}^{1}(\Omega)$ is a weak solution of (1.1).

Theorem 3.4. For $f \in L^{\infty}(\Omega)$ and for $n \geqslant 5$, suppose that

$$
|\Omega|^{2 / n}<L(n)
$$

and that at least one of following inequalities holds

$$
\|f\|_{\infty}<\frac{A(n)}{|\Omega|^{\frac{n+2}{2 n}}}, \quad \text { if } \quad|\Omega|>1,
$$

or

$$
\|f\|_{\infty}<A(n), \text { if }|\Omega| \leqslant 1 \text {, }
$$


Existence of Solutions for some Elliptic Problems with Critical Sobolev Exponents 191

where

$$
L(n)=\frac{\left[\frac{4}{n+2}\left(\frac{n-2}{n+2}\right)^{\frac{n-2}{4}}\right]^{\frac{2}{n}}-\left(\frac{n-1}{n-2} \frac{1}{\sqrt{n}}\right)^{2 \frac{n+2}{n-2}}}{\left(\frac{n-1}{n-2} \frac{1}{\sqrt{n}}\right)^{2}}
$$

and

$$
A(n)=\frac{\left(\frac{n-2}{n+2}\right)^{\frac{n^{2}-4}{8 n}}-\left(\frac{n-2}{n+2}\right)^{\frac{(n+2)^{2}}{8 n}}}{\left[\left(\frac{n-1}{n-2} \frac{1}{\sqrt{n}}\right)^{2}|\Omega|^{\frac{2}{n}}+\left(\frac{n-1}{n-2} \frac{1}{\sqrt{n}}\right)^{\frac{2(n+2)}{n-2}}\right]^{\frac{n-2}{4}}} .
$$

Then problem (1.2) has at least a weak solution if we suppose that $\partial \Omega$ is sufficiently smooth.

Proof. As in Theorem 3.3 we will consider here the problem

$$
\begin{aligned}
& \Delta u+u+|u|^{s}+f=0 \quad \text { in } \quad \Omega, \\
& u(x)=0 \quad \text { on } \quad \partial \Omega,
\end{aligned}
$$

with $1<s<\frac{n+2}{n-2}$.

Let $g(x, u)=u+|u|^{s}+f$. Then if $\|u\|_{s+1} \leqslant r,\left\|u+|u|^{s}\right\|_{\frac{s+1}{s}} \leqslant \epsilon$ and the relationship betwen $r$ and $\epsilon$ can be taken to be

$$
r|\Omega| s-1 \mid s+1+r^{s}=\epsilon
$$

As in the Theorem 3.3, problem (3.37) has a weak solution whenever there exists $x>0$ such that

$$
\left[\left(K^{2} x\right)^{s+1}+r^{s+1}\right]^{\frac{s}{s+1}} \frac{\epsilon}{r^{s}}+\|f\|_{\frac{s+1}{s}} \leqslant x .
$$

If we take

$$
r=K^{2}
$$

$K$ as in (2.5), then (3.39) becomes

$$
\left(x^{s+1}+1\right)^{\frac{s}{s+1} \epsilon}+\|f\|_{\frac{s+1}{s}} \leqslant x .
$$


Now, it is easy to see that

$$
x_{0}=\left(\frac{1}{s}\right)^{\frac{s}{s^{2}-1}} \epsilon^{-\left(\frac{1}{s-1}\right)}
$$

is a solution of (3.41) if the following two inequalities hold:

$$
\epsilon^{\frac{s+1}{s-1}}<\left(\frac{1}{s}\right)^{\frac{1}{s-1}}-\left(\frac{1}{s}\right)^{\frac{s}{s-1}}
$$

and

$$
\|f\|_{\frac{s+1}{s}}<\frac{\left(\frac{1}{s}\right)^{\frac{s}{s^{2}-1}}-\left(\frac{1}{s}\right)^{\frac{s^{2}}{s^{2}-1}}}{\epsilon^{\frac{1}{s-1}}} .
$$

Now, by (3.38), $\epsilon=|\Omega|^{\frac{s-1}{s+1}} K^{2}+K^{2 s}, K$ as in (2.5). If we take the limit when $s \rightarrow N$, in both sides of (3.42) we obtain (3.32). Then (3.32) implies that there exists $s_{1} \in R, 1<s_{1}<N$, such that for all $s \in\left(s_{1}, N\right)$ the inequality (3.42) holds. Also, by (3.33) or (3.34) there exists $s_{2}<N$ such that, for all $s \in\left(s_{2}, N\right)$ the inequality holds. Let $s_{0}=\operatorname{Max}\left\{s_{1}, s_{2}\right\}$; then for all $s \in\left(s_{0}, N\right)$ problem (3.37) has a weak solution. We may argue as in Theorem 3.3 and repeat all the formulas in (3.23) to (3.30) and obtain that there exists $u_{N} \in H_{0}^{1}(\Omega)$ a weak solution of the problem (1.2).

Remarks. If $|\Omega|<1$, Theorem 3.4 holds for $n \geqslant 4$ and in that case, for $n \geqslant 5$, (3.32) is superfluous.

I am gratefult to Dr. Yu Takeuchi who showed me that

$$
\lim _{n \rightarrow \infty} A(n)=\lim _{n \rightarrow \infty} B(n)=\lim _{n \rightarrow \infty} L(n)=\infty .
$$

Following the same argument of Theorem 3.4 we have the following

Theorem 3.5. For $f \in L^{\infty}(\Omega)$ and $n \geqslant 4$ suppose that

$$
|\lambda||\Omega|^{2 / n}<L(n)
$$

and that at least one of following inequalities holds

$$
\|f\|_{\infty}<\frac{A(|\lambda|, n)}{|\Omega|^{\frac{n+2}{2 n}}}, \text { if }|\Omega|>1
$$

or

$$
\|f\|_{\infty}<A(|\lambda|, n), \text { if }|\Omega| \leqslant 1
$$


where

$$
A(|\lambda|, n)=\frac{\left(\frac{n-2}{n+2}\right)^{\frac{n^{2}-4}{8 n}}-\left(\frac{n-2}{n+2}\right)^{\frac{(n+2)^{2}}{8 n}}}{\left[\left(\frac{n-1}{n-2} \frac{1}{\sqrt{n}}\right)^{2}|\lambda||\Omega|^{\frac{2}{n}}+\left(\frac{n-1}{n-2} \frac{1}{\sqrt{n}}\right)^{\frac{2(n+2)}{n-2}}\right]^{\frac{n-2}{4}}}
$$

\section{Then the problem}

$$
\left.\begin{array}{rlr}
\Delta u+\lambda u+|u|^{N}+f=0 & \text { in } & \Omega, \\
u(x)=0 & \text { on } & \partial \Omega,
\end{array}\right\}
$$

has at least a weak solution.

If we suppose that $f>0$ on $\Omega$ then, by the Maximum principle for the operator $\Delta$, we have that problems (1.1), (1.2) and (3.48) for $\lambda \geqslant 0$, have positive solutions.

Finally, in Theorem 3.4, if $|\Omega|$ is sufficiently small then $\epsilon=|\Omega|^{s-1}+r^{s}$ is small too $\left(r=K^{2}\right)$. Then inequality (3.41) holds for some $x>\|f\|_{\infty}$. We conclude by saying that Problem (1.2) has a weak solution if $|\Omega|$ is sufficiently small.

\section{References}

[1] Berger, M. Nonlinearity and Functional Analysis. Academic Press, 1977.

[2] Brezis, H. and Niremberg, L. Positive Solutions of Nonlinear Elliptic Equations involving Critical Sobolev Exponents. Comm. Pure Appl. Math. 36(1983), 437-477.

[3] Chow, S. and Hale, J. Methods of Bifurcation Theory. Springer-Verlag, 1982.

[4] Escobar, J. Positive Solutions for some semilinear Elliptic Equations with Critical Sobolev Exponents. Comm. Pure Appl. Math. 50(1987), 623-657.

[5] Krasnosel'skii, M. Topological Methods in the Theory of Nonlinear Integral Equations. Pergamon Press, 1964.

[6] Lee, J. and Parker, T. The Yamabe Problem. Bull. Amer. Math. Soc. 17(1987), 37-91.

Recibido: 5 de julio de 1988 .

Mario Zuluaga

Departamento de Matemáticas Universidad Nacional de Colombia 